\title{
Use of a pH-sensitive polymer in a microextraction and preconcentration method directly combined with high-performance liquid chromatography
}

\author{
Raúl González-Martín a, Idaira Pacheco-Fernández a,b, Binoy Maitic ${ }^{c}$, Juan H. Ayala a \\ Ana M. Afonso ${ }^{a}$, David Díaz Díaz ${ }^{\mathrm{c}, \mathrm{d}, \mathrm{e}, *}$, Verónica Pino ${ }^{\mathrm{a}, \mathrm{b}, *}$ \\ a Departamento de Química, Unidad Departamental de Química Analítica, Universidad de La Laguna (ULL), Tenerife, 38206, Spain \\ ${ }^{\mathrm{b}}$ Instituto Universitario de Enfermedades Tropicales y Salud Pública de Canarias, Universidad de La Laguna (ULL), Tenerife, 38206, Spain \\ ${ }^{c}$ Institut für Organische Chemie, Universität Regensburg, Regensburg, 93053, Germany \\ d Departamento de Química Orgánica, Universidad de La Laguna (ULL), Tenerife, 38206, Spain \\ e Instituto Universitario de Bio-Orgánica “Antonio González", Tenerife, 38206, Spain
}

\section{A R T I C L E I N F O}

\section{Article history:}

Received 6 December 2019

Revised 9 January 2020

Accepted 21 January 2020

Available online 23 January 2020

\section{Keywords:}

$\mathrm{pH}$-sensitive polymer

Hydrogel

Microextraction

Liquid chromatography

Polycyclic aromatic hydrocarbons

Urine

\begin{abstract}
A B S T R A C T
A pH-sensitive polymer based on the poly(styrene-alt-maleic anhydride) co-polymer serves as basis to develop a microextraction method (pH-HGME) in direct combination with high-performance liquid chromatography (HPLC) and fluorescence detection (FD) for the determination of seven organic compounds, including three polycyclic aromatic hydrocarbons (PAHs), three monohydroxylated PAHs and one alkylphenol, in urine. The method bases on the structural modification of the pH-sensitive polymer in the aqueous sample at a high $\mathrm{pH}$ value, followed by the formation and insolubilization of a hydrogel containing the preconcentrated analytes by decreasing the $\mathrm{pH}$, and the direct injection of the hydrogel-rich phase in the HPLC-FD system. The optimization of the main variables permitted the selection of low amounts of aqueous sample $(10 \mathrm{~mL})$, which was mixed with $10 \mathrm{mg}$ of co-polymer also present in a low volume $(150 \mu \mathrm{L})$ of concentrated $\mathrm{NaOH}$. The method further requires the addition of $200 \mu \mathrm{L}$ of concentrated $\mathrm{HCl}$, 3 min of stirring, and 15 min of centrifugation. This pH-HGME-HPLC-FD method presented low limits of detection, ranging from $0.001 \mu \mathrm{L} \mathrm{L}^{-1}$ to $0.09 \mu \mathrm{g} \mathrm{L} \mathrm{L}^{-1}$ in ultrapure water, average relative recoveries of $96.9 \%$ for the concentration level of $0.60 \mu \mathrm{g} \mathrm{L}^{-1}$, and enrichment factors between 1.50 and 17.7. The proposed method also exhibited high precision, with intermediate relative standard deviations lower than $16 \%$ for a concentration level of $0.60 \mu \mathrm{g} \mathrm{L}^{-1}$. The developed pH-HGME-HPLC-FD method performed adequately when analyzing two human urine samples provided by a non-smoker male and a smoker female, respectively. One of the target analytes (2-hydroxynaphthalene) was quantified in both samples using the standard addition method, with a predicted concentration of $7.3 \pm 0.4 \mu \mathrm{g} \mathrm{L}{ }^{-1}$ in the non-smoker male urine and $19.3 \pm 0.6 \mu \mathrm{g} \mathrm{L}^{-1}$ in the smoker female urine.
\end{abstract}

(c) 2020 Elsevier B.V. All rights reserved.

\section{Introduction}

Polymer hydrogels are three-dimensional networks made of polymers that have a high water content [1]. During the last decades, these hydrogels have been mainly used as cell carriers and structural scaffolds in tissue engineering applications due to

\footnotetext{
* Corresponding authors.

E-mail addresses: raul.gonzalez.26@ull.edu.es (R. González-Martín), ipacheco@ull.edu.es (I. Pacheco-Fernández), Maiti.Binoy@chemie.uni-regensburg.de (B. Maiti), jayala@ull.edu.es (J.H. Ayala), aafonso@ull.edu.es (A.M. Afonso), ddiazdiaz@ull.edu.es (D.D. Díaz), veropino@ull.edu.es (V. Pino).
}

their structural similarities to the natural extracellular matrix [2,3]. Moreover, applications in many other fields (e.g., microfluidics, optics, soft robotics...) are also known [4]. Many of these hydrogels are refereed as smart materials due to their ability to respond to various external stimuli such as $\mathrm{pH}$, temperature, chemical additives, ionic strength, light, electric, and magnetic field [57]. Among these gels, pH-sensitive hydrogels constitute versatile platforms with controllable structural and volume phase transitions, which have been commonly exploited for the encapsulation and sustained release of active compounds upon specific changes in $\mathrm{pH}[8-10]$.

Despite the potential of stimuli-responsive polymer hydrogels as sorbent materials, their use in analytical extraction methods has 
been barely explored. In this context, Bahram et al. were the first to propose the use of a pH-sensitive polymer as extraction material in an analytical method [11,12]. The polymer was assessed for the extraction of malachite green [11] and copper [12] from water samples, followed by their respective determinations using spectrophotometric techniques.

The application of a temperature-sensitive polymer for the determination of phenols in moat river samples has also been reported [13]. In the study, the polymer was added to the sample and then the mixture was heated to perform the hydrophilichydrophobic phase transition of the polymer. However, the addition of a salt was required for the phase separation, followed by the elution of the analytes retained in the hydrophobic polymer with an organic solvent prior to the injection is a gas chromatograph.

Furthermore, the use of a $\mathrm{pH} /$ thermo dual-responsive hydrogel for the determination of fluoroquinolones in water and food samples has been reported by Yu et al. [14]. Despite the extraction and desorption of the analytes could be controlled by specific changes in both the $\mathrm{pH}$ and the temperature, the experimental procedure required a desorption step followed by a reconstitution step prior to the final determination.

More recently, it was described the use of a non-stimuliresponsive hydrogel based on polyvinyl alcohol/pectin for the extraction of steroidal hormones from aqueous samples [15]. In this case, the hydrogel was used as sorbent to prepare a miniaturized solid-phase extraction device. Thus, the method required activation of the material, loading, washing and desorption steps, together with a final derivatization step.

Despite the success of these strategies, all of them require an additional back-extraction or desorption step before the analytical determination technique, leading to time-consuming and tedious methods. This, in turns, avoids the consideration of the method within the green analytical chemistry requirements [16].

Therefore, the aim of this study is the incorporation of a $\mathrm{pH}-$ sensitive polymer in a simple microextraction and preconcentration method that ensures, for the first time, a direct combination with liquid chromatography, avoiding any type of desorption/backextraction step. Furthermore, this study intends the applicability of the method to complex biological samples in contrast to previous studies reporting the use of stimuli-responsive polymers, which are mainly devoted to the analysis of environmental waters [11-15]. Thus, human urine samples, barely analyzed with liquid-phase microextraction methods [17], were tested as matrix. The target analytes were a group of seven organic compounds with demonstrated carcinogenic and endocrine disruptor effects: three polycyclic aromatic hydrocarbons - PAHs -, three monohydroxylated PAHs - OHPAHs - and one alkylphenol.

\section{Experimental}

\subsection{Chemicals, reagents, materials, and samples}

Styrene (>99.9\%), maleic anhydride (99\%) and azobisisobutyronitrile (AIBN) - used to synthesize poly(styrene-alt-maleic anhydride) - together with diethyl ether ( $\geq 99.7 \%)$ and hexadeuterated dimethyl sulfoxide (DMSO- $d_{6}$ ), were supplied by Sigma-Aldrich (Steinheim, Germany). N,N-dimethylformamide (DMF, $\geq 99.5 \%$ ) was purchased from Merck KGaA (Darmstadt, Germany), and acetonitrile $(\mathrm{ACN})$ HiPerSolv Chromanorm ${ }^{\circledR}$ LC grade was provided by VWR (Llinars del Vallés, Spain).

The studied analytes were three PAHs: fluorene (Flu, 98\%), fluoranthene (Ft, 98\%) and benz[a]anthracene (BaA, 99\%), three $\mathrm{OH}$ PAHs: 2-hydroxynaphthalene (2OHnap, 99.9\%), 2-hydroxyfluorene (2OHflu, 98\%) and 2-hydroxyphenanthrene (2OHphe, standard solution of $10 \mathrm{mg} \mathrm{L}^{-1}$ in $\mathrm{ACN}$ ), and one alkylphenol: 4-nonylphenol
(nNP, 99\%). PAHs were supplied by Sigma-Aldrich, while $\mathrm{OH}-$ PAHs and nNP were provided by Dr. Ehrenstorfer GmbH (Augsburg, Germany). Table S1 of the Electronic Supplementary Material (ESM) contains the chemical structures and several physicochemical properties of the analytes. Individual standard solutions of all the analytes (except for 2OHphe) were prepared in $\mathrm{ACN}$ at the following concentrations: $560 \mathrm{mg} \mathrm{L}^{-1}$ for 20Hnap, $600 \mathrm{mg} \mathrm{L}^{-1}$ for 2OHflu, $4100 \mathrm{mg} \mathrm{L}^{-1}$ for Flu, $2600 \mathrm{mg} \mathrm{L}^{-1}$ for Ft, $2160 \mathrm{mg} \mathrm{L}^{-1}$ for $\mathrm{BaA}$ and $1003 \mathrm{mg} \mathrm{L}^{-1}$ for nNP. Intermediate standard solutions containing all the analytes in ACN were prepared at $5 \mu \mathrm{g} \mathrm{L}^{-1}$, $100 \mu \mathrm{g} \mathrm{L}^{-1}, 1000 \mu \mathrm{g} \mathrm{L}^{-1}$ and $5000 \mu \mathrm{g} \mathrm{L}^{-1}$. All these standard solutions were kept protected from light and refrigerated at $4{ }^{\circ} \mathrm{C}$. Working standard solutions were prepared in ultrapure water using these intermediate solutions.

Hydrochloric acid $(37 \%, v / v)$, supplied by Honeywell Fluka ${ }^{\mathrm{TM}}$ (Seetze, Germany), and sodium hydroxide ( $\geq 99.5 \%$ ), purchased from Sigma-Aldrich, were used in the microextraction method. Solutions of these reagents were prepared in ultrapure water at the following concentrations: $2.0 \mathrm{M}$ and $3.0 \mathrm{M}$ for $\mathrm{NaOH}$; and $2.5 \mathrm{M}$, 3.8 M, 5.0 M, 6.3 $\mathrm{M}$ and 7.5 $\mathrm{M}$ for $\mathrm{HCl}$.

The synthesis of poly(styrene-alt-maleic anhydride) was performed using $20 \mathrm{~mL}$ clear glass vials with screw caps and polytetrafluoroethylene (PTFE) septa purchased from Supelco (Bellefonte, PA, USA), stir bars $(15 \times 4.5 \mathrm{~mm})$ and $0.22 \mu \mathrm{m}$ Millipore filters supplied by Sigma-Aldrich. Pyrex ${ }^{\circledR}$ (Staffordshire, United Kingdom) centrifuge tubes of $15 \mathrm{~mL}$ (9.5 cm L $\times 2 \mathrm{~cm}$ O.D.) were used for the development of the microextraction method.

Ultrapure water with a resistivity of $18.2 \mathrm{M} \Omega \mathrm{cm}$, obtained from a Milli-Q water purification system (Watford, United Kingdom), and ACN HiPerSolv Chromanorm ${ }^{\circledR}$ grade LC were used for the preparation of mobile phases. They were filtered through $0.22 \mu \mathrm{m}$ Millipore filters. A $50 \mu \mathrm{L}$ Hamilton syringe (Reno, Nevada, USA) was used for the injection in the HPLC system.

Two urine samples were analyzed in this study, which were provided by two healthy volunteers: a non-smoker male and a smoker female, who previously signed an individual informed consent.

\subsection{Instrumentation and equipment}

The co-polymerization reaction was carried out using a metallic block placed on a heating plate from $P$ Selecta ${ }^{\circledR}$ (Barcelona, Spain) with temperature and magnetic stirring control. A vacuum pump VP 2 Autoyac from Vacuubrand (Wertheim, Germany) was used to filter and dry the co-polymer. A 5702 centrifuge from Eppendorf $^{\mathrm{TM}}$ (Hamburg, Germany) and a vortex agitation system from Velp ${ }^{\circledR}$ Scientifica (Usmate, Italy) were used in the microextraction method.

Poly(styrene-alt-maleic anhydride) co-polymer was characterized by one-dimensional proton nuclear magnetic resonance spectroscopy ( $\left.{ }^{1} \mathrm{H}-\mathrm{NMR}\right)$, using an Avance ${ }^{\mathrm{TM}}$ NMR spectrometer (500 MHz) from Bruker (Massachusetts, USA).

The chromatographic separation was performed using a 1260 Infinity HPLC system coupled with a 1260 Infinity multichannel fluorescence detector (FD) from Agilent Technologies (Santa Clara, CA, USA). The chromatographic system was equipped with a Rheodyne 7725i injection valve with a $5 \mu \mathrm{L}$ loop supplied by Supelco. The chromatographic column was an InfinityLab Poroshell 120 ECC18 (150 mm $\times 4.6 \mathrm{~mm} \times 4 \mu \mathrm{m}, 12 \mathrm{~nm}$ of pore size) purchased from Agilent Technologies and protected by a Pelliguard LC-C18 guard column from Supelco.

The software Statgraphics ${ }^{\circledR}$ Centurion XV was used for the statistical calculations of the experimental design. Excel (Microsoft Office, v. 2016) was used for the remaining calculations. 


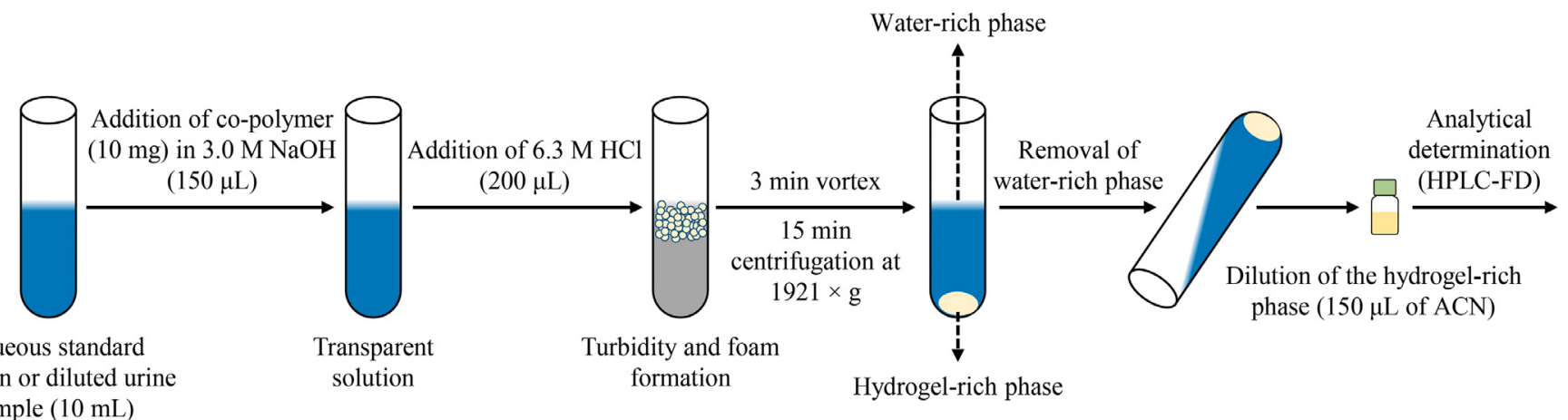

Fig. 1. Scheme of the microextraction procedure with a pH-sensitive polymer based on poly(styrene-alt-maleic anhydride) under optimum conditions.

\subsection{Procedures}

\subsubsection{Synthesis of poly(styrene-alt-maleic anhydride)}

Poly(styrene-alt-maleic anhydride) was synthesized according to a procedure previously described in the literature [18] with slight modifications. Briefly, styrene ( $4.9 \mathrm{mmol}, 568 \mu \mathrm{L})$, maleic anhydride (4.9 mmol, $485 \mathrm{mg}$ ) and AIBN (0.04 mmol, $6.5 \mathrm{mg}$ ) were mixed with DMF $(4 \mathrm{~mL})$ in a $20 \mathrm{~mL}$ sealed glass vial. The mixture was purged with dried $\mathrm{N}_{2}$ for $20 \mathrm{~min}$. The co-polymerization reaction was carried out at $70{ }^{\circ} \mathrm{C}$ for $14 \mathrm{~h}$ followed by cooling in an ice-water bath.

The obtained co-polymer was purified by re-precipitation with diethyl ether $(\times 5)$. The product was filtered through a $0.22 \mu \mathrm{m}$ filter and dried under high vacuum $\left(7.00 \cdot 10^{4} \mathrm{~N} \mathrm{~m}^{-2}\right)$ at room temperature for $8 \mathrm{~h}$. Figure S1 of the ESM includes a scheme of the co-polymerization reaction together with the synthetic procedure. Finally, the structure of the co-polymer was confirmed by ${ }^{1} \mathrm{H}-\mathrm{NMR}$ in DMSO- $d_{6}$. The co-polymer has an average molecular weight of $59,130 \mathrm{~g} \mathrm{~mol}^{-1}$ as previously reported in the literature depending on the polydispersity index [18].

\subsubsection{HPLC-FD method}

The chromatographic separation of the analytes was accomplished using a mobile phase composed of ACN and ultrapure water at a constant flow of $1 \mathrm{~mL} \mathrm{~min}^{-1}$. The elution gradient started at $60 \%(\mathrm{v} / \mathrm{v})$ of $\mathrm{ACN}$, being then increased to $62 \%(\mathrm{v} / \mathrm{v})$ in $5 \mathrm{~min}$, and then up to $100 \%(\mathrm{v} / \mathrm{v})$ in $0.5 \mathrm{~min}$, while kept at this value for $6.5 \mathrm{~min}$.

The fluorescence program used for the detection of the analytes was optimized, to establish those excitation $\left(\lambda_{\text {ex }}\right)$ and emission $\left(\lambda_{\mathrm{em}}\right)$ wavelengths ensuring maximum sensitivity for each analyte. The $\lambda_{\text {em }}$ was kept constant at each channel, while the $\lambda_{\text {ex }}$ was changed at different chromatographic times during the separation depending on the analyte. The optimum fluorescence program is shown in Table S2 of the ESM.

\subsubsection{Microextraction procedure using the $\mathrm{pH}$-sensitive polymer based on poly(styrene-alt-maleic anhydride)}

Under optimized conditions, $10 \mathrm{mg}$ of poly(styrene-alt-maleic anhydride) were dissolved in $150 \mu \mathrm{L}$ of $3.0 \mathrm{M} \mathrm{NaOH}$ to accomplish the hydrolysis of the co-polymer. Then, the co-polymer (in the basic medium) was added to a centrifuge tube containing $10 \mathrm{~mL}$ of an aqueous standard solution or a diluted urine sample (spiked and non-hydrolyzed, spiked and hydrolyzed, or non-spiked and hydrolyzed, depending on the specific experiment). After manual stirring for a few seconds, the polymer solubilizes in the aqueous medium. Then, $200 \mu \mathrm{L}$ of $6.3 \mathrm{M} \mathrm{HCl}$ were added and a turbid and foamy solution was obtained, indicating the formation and insolubilization of the hydrogel. The application of vortex for $3 \mathrm{~min}$ favored the interactions of the analytes with the insolubilized hy- drogel. Subsequently, the mixture was centrifuged for $15 \mathrm{~min}$ at $1921 \times g$ to promote the separation of the phases. After centrifugation, a new hydrogel-rich phase forms at the bottom of the tube. The water-rich phase was discarded by manual decantation and the hydrogel-rich phase containing the analytes was dissolved in $150 \mu \mathrm{L}$ of ACN prior to the injection in the HPLC system.

Fig. 1 shows a scheme of the experimental procedure of the $\mathrm{pH}$ HGME method under optimized conditions.

\subsubsection{Hydrolysis of urine samples}

Sample collection of the urine samples took place in the early morning, followed by an enzymatic hydrolysis before the analysis [17]. Briefly, the hydrolysis required the addition of $15 \mathrm{~mL}$ of urine to a centrifuge tube containing $2 \mathrm{~mL}$ of acetic acid/sodium acetate buffer solution at $\mathrm{pH} 5$, followed by the addition of $50 \mu \mathrm{L}$ of the $\beta$-glucoronidase/arylsulfatase enzyme. Incubation of samples required $24 \mathrm{~h}$ at $37^{\circ} \mathrm{C}$, to ensure accomplishment of the hydrolysis reaction.

In all cases, it was performed a dilution of $1 \mathrm{~mL}$ of the urine samples (non-hydrolyzed or hydrolyzed) up to $10 \mathrm{~mL}$ with ultrapure water before the analysis.

\section{Results and discussion}

\subsection{Generation of the $p H$-sensitive hydrogel using poly(styrene-alt-maleic anhydride)}

The polymer used to generate a $\mathrm{pH}$-sensitive hydrogel must have acidic or basic functional groups in the polymeric backbone that lead to structural changes if an exchange of protons is induced [10]. Given these requirements, the $\mathrm{pH}$-sensitive polymer based on poly(styrene-alt-maleic anhydride) was selected in this study. The structure of the co-polymer was confirmed by ${ }^{1} \mathrm{H}-\mathrm{NMR}$ analysis, which provided spectroscopic data identical to those reported in the literature [19], as shown in Figure S2 of the ESM.

As a preliminary study, the possible modifications in the copolymer backbone that may allow the formation of a $\mathrm{pH}$-sensitive hydrogel were elucidated, with the aim of assessing its applicability as extraction material in a microextraction method. In this sense, the solubility of the co-polymer was evaluated at different $\mathrm{pH}$ values. It was observed that poly(styrene-alt-maleic anhydride) was insoluble in ultrapure water $(\mathrm{pH}=5.5)$, but soluble when adding $\mathrm{NaOH}(\mathrm{pH} \sim 10)$. This change in the solubility is due to the basic hydrolysis of the anhydride functional group, leading to the formation of two carboxylate groups. Subsequently, the addition of $\mathrm{HCl}$ provokes a cloudy solution due to the generation and insolubilization of a $\mathrm{pH}$-sensitive hydrogel. The protonation of the carboxylate groups after such addition of $\mathrm{HCl}$ resulted in the formation of poly(styrene-alt-maleic diacid), which is water insoluble. 
<smiles>CCC1C(=O)OC(=O)C1CC(c1ccccc1)c1ccccc1</smiles>

water-insoluble poly(styrene-alt-maleic) anhydride

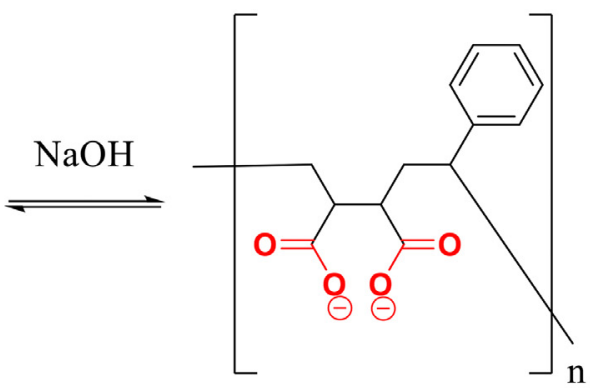

water-soluble poly(styrene-alt-maleic) dicarboxylate

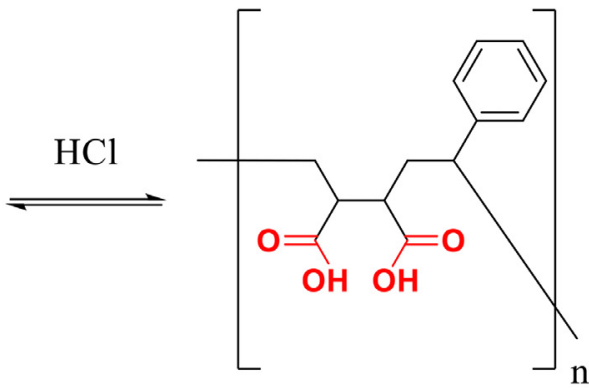

water-insoluble poly(styrene-alt-maleic) diacid

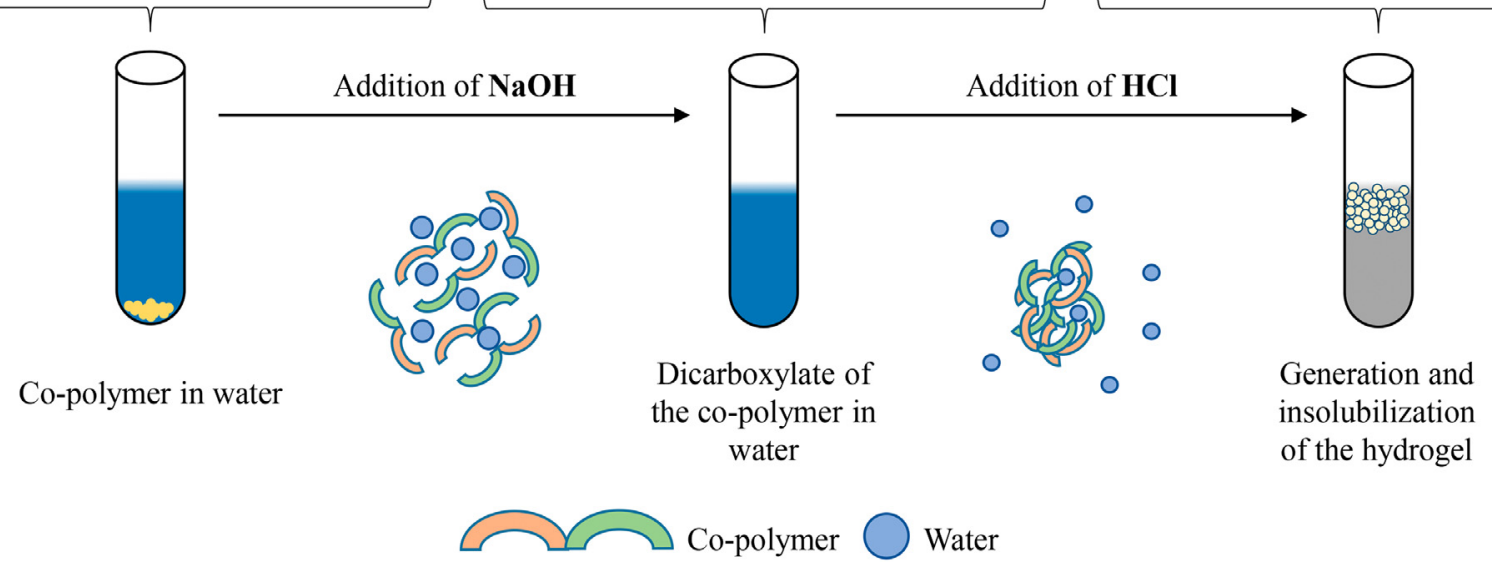

Fig. 2. Generation of a pH-sensitive hydrogel based on the structural modifications of the poly(styrene-alt-maleic anhydride) co-polymer.

Fig. 2 includes a scheme of the formation of the $\mathrm{pH}$-sensitive hydrogel based on the structural modifications of the poly(styrenealt-maleic anhydride) co-polymer.

\subsection{Development of a microextraction method using the $p H$-sensitive polymer}

\subsubsection{Assessing the applicability of the $\mathrm{pH}$-sensitive polymer as} extraction material in combination with HPLC-FD

The $\mathrm{pH}$-sensitive polymer based on poly(styrene-alt-maleic anhydride) was tested for the extraction of different organic compounds from waters, including three PAHs (Flu, Ft and BaA), three OH-PAHs (2OHnap, 2OHflu and 2OHphe) and one alkylphenol (nNP), using HPLC-FD.

The adequate separation of the analytes by HPLC-FD was achieved using the chromatographic conditions described in Section 2.3.2. Fig. 3(A) shows a representative chromatogram obtained after the injection of a standard (in ACN) containing $50 \mu \mathrm{g} \mathrm{L}^{-1}$ of the analytes. Table S3 of the ESM includes the quality analytical parameters of the HPLC-FD method.

In order to assess the compatibility of the $\mathrm{pH}$-sensitive polymer with the HPLC-FD system, its effect on the chromatographic separation for the target analytes, and its extraction capability, the polymer was used in a microextraction method under random preliminary conditions. Thus, $3.5 \mathrm{mg}$ of the co-polymer - dissolved in $100 \mu \mathrm{L}$ of $2.0 \mathrm{M} \mathrm{NaOH}$ - were added to $10 \mathrm{~mL}$ of an aqueous standard containing $5 \mu \mathrm{g} \mathrm{L} \mathrm{L}^{-1}$ of the analytes. A hydrogel was formed by adding $500 \mu \mathrm{L}$ of $\mathrm{HCl}(2.0 \mathrm{M})$. The mixture was subjected to vortex stirring for $3 \mathrm{~min}$ followed by centrifugation for $10 \mathrm{~min}$ at $1921 \times \mathrm{g}$, forming a hydrogel-rich phase at the bottom of the tube. The solubility of the hydrogel-rich phase in different HPLCcompatible solvents was tested, with ACN $(250 \mu \mathrm{L})$ the most ade- quate solvent. At this point, it was necessary to verify if the optimum chromatographic conditions described in Section 2.3.2 were also favorable for the hydrogel-rich phase, while ensuring its compatibility with the HPLC column. Indeed, the initial composition of the mobile phase $(60 \%$ of $A C N)$ was enough to ensure the complete solubility of the polymer, thus avoiding the retention of the polymer into the stationary phase. Taking advantage of this compatibility, the presence of the analytes in the hydrogel-rich phase could be verified by injecting directly this diluted hydrogel-rich phase in the HPLC-FD system. Fig. 3(B) shows a representative chromatogram. As it can be observed, there is a non-interfering signal coming from the polymer at the beginning of the chromatogram, which does not affect the determination of the analytes. Furthermore, in order to ensure that the direct injection of the hydrogelrich phase was not harmful for the chromatographic column, there was an exhaustive control of the pressure and performance during all the experiments. In addition, blanks were carried out daily to verify that the polymer eluted and did not keep retained in the stationary phase. Additionally, a guard-column was always utilized (see Section 2.2).

The tool used to screen the efficiency of the polymer to preconcentrate the target analytes under these preliminary conditions was the enrichment factor $\left(\mathrm{E}_{\mathrm{F}}\right)$. The $\mathrm{E}_{\mathrm{F}}$ values were calculated as the ratio of the predicted concentration (calculated with the calibration curves of the HPLC-FD method - Table S3 of the ESM and using peak area obtained after the injection of the hydrogelrich phase), and the initial concentration of the aqueous standard subjected to the entire microextraction approach (using these preliminary conditions). The $\mathrm{E}_{\mathrm{F}}$ values ranged between 0.4 for 20Hnap and 10.8 for $\mathrm{BaA}$, which evidence the competence of the method to extract and preconcentrate several of the target analytes. Furthermore, these preliminary studies demonstrated the ability of the 


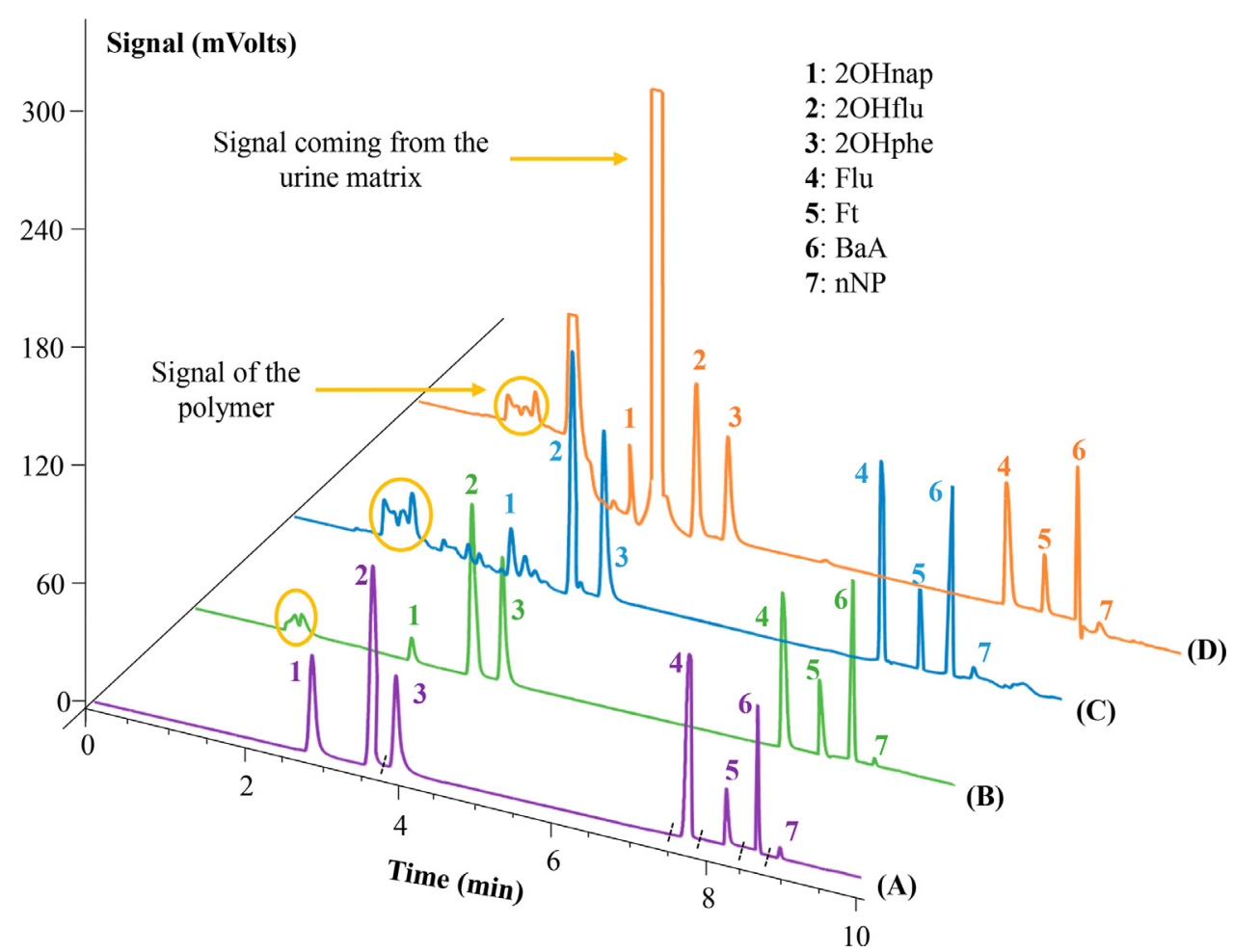

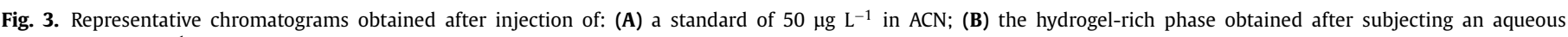

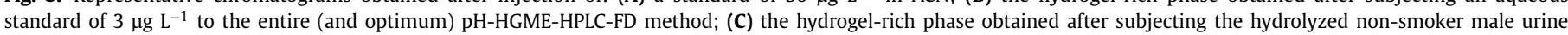

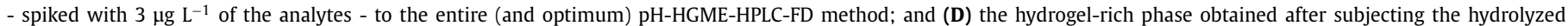

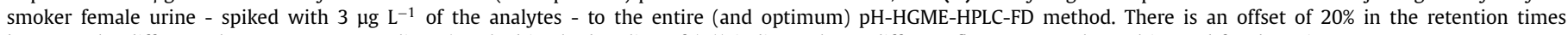
between the different chromatograms. Dot lines (marked in the baseline of $(\mathbf{A})$ ) indicate that a different fluorescence channel is used for detection.

pH-sensitive polymer to be included in a microextraction method (pH-HGME method) followed by its easy coupling with HPLC, without requiring any back-extraction step in contrast to other methods with hydrogels reported up to date [13-15]. In addition to this, the microextraction procedure using the $\mathrm{pH}$-sensitive polymer based on poly(styrene-alt-maleic anhydride) is the first application in combination with chromatography, thus maximizing its further applicability.

\subsubsection{Optimization of the $p H-H G M E$ method}

Several experimental variables were fixed initially to assess which were the main variables that exert an influence on the microextraction procedure using the $\mathrm{pH}$-sensitive polymer. Thus, the volume of the aqueous sample (or aqueous standard solution) was set at $10 \mathrm{~mL}$, the vortex stirring was fixed at $3 \mathrm{~min}$, while using $15 \mathrm{~min}$ of centrifugation at $1921 \times \mathrm{g}$. Moreover, $150 \mu \mathrm{L}$ was the minimum amount of $\mathrm{ACN}$ required to dissolve the hydrogel-rich phase prior to the injection in HPLC-FD. This minimum amount avoids further losses of preconcentration.

The extraction efficiency of the pH-HGME method was evaluated using the peak area obtained for each analyte in the HPLCFD. The studied variables were the amount of co-polymer initially added to the aqueous solution, the volume and concentration of $\mathrm{NaOH}$ used to carry out the initial hydrolysis of the co-polymer, and the volume and concentration of $\mathrm{HCl}$ employed for the formation of the hydrogel. It was observed that the variation of the volume and concentration of $\mathrm{NaOH}$ did not imply any significant effect in the performance of the microextraction method, thus fixing these variables to ensure a basic medium that required a volume of $\mathrm{NaOH}$ as low as possible $(150 \mu \mathrm{L}$ of $3.0 \mathrm{M} \mathrm{NaOH})$. Furthermore, the volume of $\mathrm{HCl}$ was fixed as the minimum value capable of generating the hydrogel, to avoid any possible loss of preconcentration $(200 \mu \mathrm{L}$ of $\mathrm{HCl})$. Therefore, taking into account the properties of the $\mathrm{pH}$-sensitive polymer together with the results of the preliminary study, the $\mathrm{HCl}$ concentration and the amount of co-polymer were selected as the two main factors to optimize.

The optimization of the two main factors was performed using a two-variable Doehlert experimental design [20]. The number of experiences was calculated following Eq. (1):

$N=k^{2}+k+C_{0}$

where $N$ is the overall number of experiments, $k$ is the number of variables to optimize, and $C_{0}$ is the number of experiments repeating the center point. In this case, the experimental design required 9 experiments, considering the center point in triplicate.

Attending to the preliminary studies, the $\mathrm{HCl}$ concentration was the factor that exerted the highest influence on the pH-HGME method. Therefore, the $\mathrm{HCl}$ concentration was evaluated at $5 \mathrm{lev}$ els, while the amount of co-polymer was studied at 3 levels. Table S4 of the ESM includes the Doehlert matrix for the current study. The $\mathrm{HCl}$ concentration was studied from $2.5 \mathrm{M}$ to $7.5 \mathrm{M}$ (to ensure a high acidic medium able to quantitatively form the hydrogel), and the amount of co-polymer ranged between $3.0 \mathrm{mg}$ and $10 \mathrm{mg}$ (trying in all cases to minimize this amount, thus ensuring a green analytical microextraction method).

Response surfaces of the experimental design followed this second-order polynomial response equation (Eq. (2)):

$$
\begin{aligned}
Y= & k+A \cdot\left[\mathrm{C}_{\mathrm{HCl}}\right]+B \cdot\left[\mathrm{m}_{\mathrm{co}-\text { polymer }}\right]+\mathrm{AA} \cdot\left[\mathrm{C}_{\mathrm{HCl}}\right]^{2}+\mathrm{AB} \cdot\left[\mathrm{C}_{\mathrm{HCl}}\right] \\
& \cdot\left[\mathrm{m}_{\text {co-polymer }}\right]+\mathrm{BB} \cdot\left[\mathrm{m}_{\mathrm{co}-\text { polymer }}\right]^{2}
\end{aligned}
$$

where $Y$ is the peak area obtained as response in the experimental design, $k$ is a constant, $A$ is the coefficient associated with the $\mathrm{HCl}$ concentration, and $B$ is the coefficient for the amount of copolymer. Each analyte presents a different response surface defined by Eq. (2), depending on the effect of the evaluated variables 
OH-PAHs (2OHnap)

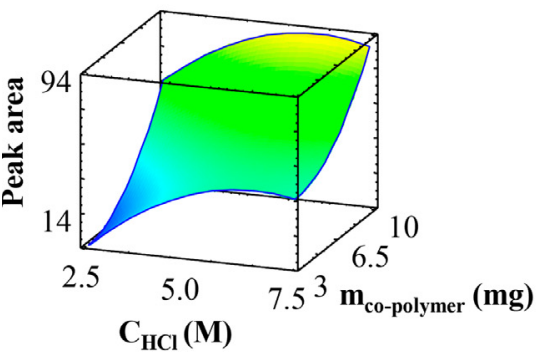

PAHs (Ft)

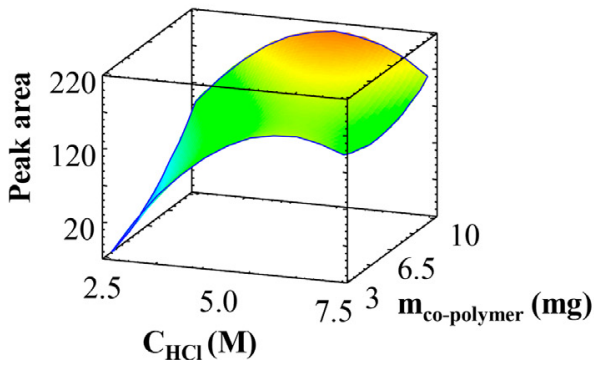

Alkylphenol (nNP)

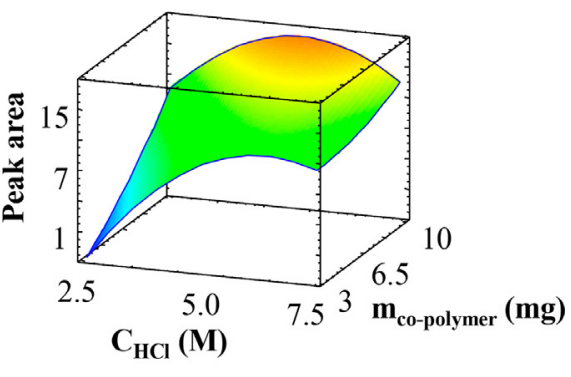

Fig. 4. Representative response surfaces for the variables optimized with the Doehlert experimental design.

Table 1

Several quality analytical parameters of the entire pH-HGME-HPLC-FD method in ultrapure water. LODs and LOQs of the chromatographic method (HPLC-FD) are also included with comparative purposes.

\begin{tabular}{|c|c|c|c|c|c|c|c|c|}
\hline \multirow{2}{*}{ Analyte } & \multirow{2}{*}{$\begin{array}{l}\text { Calibration } \\
\text { range }\left(\mu \mathrm{g} \mathrm{L}^{-1}\right)\end{array}$} & \multirow{2}{*}{$\begin{array}{l}\text { (Slope } \pm \\
\text { SD }^{\mathrm{a}} \text { ) }\end{array}$} & \multirow{2}{*}{$\mathrm{R}^{\mathrm{b}}$} & \multirow{2}{*}{$\mathrm{S}_{\mathrm{y} / \mathrm{x}}{ }^{\mathrm{c}}$} & \multicolumn{2}{|l|}{$\operatorname{LOD}^{\mathrm{d}}\left(\mu \mathrm{g} \mathrm{L}^{-1}\right)$} & \multicolumn{2}{|l|}{$\mathrm{LOQ}^{\mathrm{e}}\left(\mu \mathrm{g} \mathrm{L}^{-1}\right)$} \\
\hline & & & & & pH-HGME-HPLC-FD & HPLC-FD & pH-HGME-HPLC-FD & HPLC-FD \\
\hline 20Hnap & $0.3-3$ & $17 \pm 1$ & 0.998 & 1.3 & 0.03 & 0.090 & 0.1 & 0.30 \\
\hline 2OHflu & $0.1-3$ & $138 \pm 2$ & 0.999 & 4.7 & 0.001 & 0.010 & 0.003 & 0.030 \\
\hline 2OHphe & $0.1-3$ & $117 \pm 2$ & 0.999 & 4.8 & 0.004 & 0.060 & 0.01 & 0.20 \\
\hline Flu & $0.1-3$ & $117 \pm 2$ & 0.999 & 4.9 & 0.02 & 0.20 & 0.07 & 0.70 \\
\hline $\mathrm{Ft}$ & $0.1-3$ & $38 \pm 1$ & 0.999 & 2.5 & 0.003 & 0.060 & 0.01 & 0.20 \\
\hline $\mathrm{BaA}$ & $0.1-3$ & $93 \pm 4$ & 0.997 & 8.7 & 0.001 & 0.030 & 0.003 & 0.10 \\
\hline nNP & $0.5-5$ & $3.1 \pm 0.1$ & 0.998 & 0.4 & 0.09 & 0.75 & 0.3 & 2.5 \\
\hline
\end{tabular}

a Standard deviation of the slope within the calibration range $(n=6)$ for a significance level of $5 \%$.

b Correlation coefficient.

c Standard deviation of the residuals.

d Limit of detection determined by decreasing the concentration of the standards until a S/N ratio of 3 was obtained.

e Limit of quantification, estimated as 10/3 times the LOD, and experimentally verified by applying the method to aqueous standards at the predicted concentrations.

on their extraction efficiency. Table S5 of the ESM shows the coefficients of the polynomial response equation for all the tested analytes, together with the regression parameters of the model and the recommended optimum values for the two variables. It is important to highlight that the coefficients of determination $\left(\mathrm{R}^{2}\right)$ ranged from $91.8 \%$ for 20 Hnap to $99.5 \%$ for BaA.

Fig. 4 includes some representative response surfaces, corresponding to one analyte of each of the groups of compounds studied. It can be observed that there are not significant differences between the response surfaces for each group of analytes. The maximum in all cases is observed when using the highest amount of co-polymer evaluated $(10 \mathrm{mg})$, and $\mathrm{HCl}$ concentrations ranging from $5.5 \mathrm{M}$ to $6.3 \mathrm{M}$ depending on the analyte. Given the fact that there are important differences between the peak areas obtained with the optimum $\mathrm{HCl}$ concentration and those obtained when concentrations close to the optimum value are used, a compromise solution was required. In this sense, a value of $6.3 \mathrm{M}$ was chosen as the optimum $\mathrm{HCl}$ concentration of the pH-HGME method in order to benefit 20Hnap, which is the analyte with the lowest enrichment factor. In order to ensure that this selection was not accompanied by a significant loss on the extraction efficiency for the remaining analytes, the variation on the peak area (in percentage) when using the optimum $\mathrm{HCl}$ concentration for each analyte or the compromise value $(6.3 \mathrm{M})$ was calculated. The study was performed by comparing the responses obtained after applying the Eq. (2) using those $\mathrm{HCl}$ concentration values and $10 \mathrm{mg}$ of co-polymer. Table S6 of the ESM shows that the differences were lower than $4 \%$, which is a small rate considering the positive effect on the extraction efficiency of 20Hnap if the selected acid concentration is used.
Regarding the amount of co-polymer, Fig. 4 shows that the peak areas increased with the amount of co-polymer for all the analytes, having the maximum with $10 \mathrm{mg}$. These results also suggested that if the amount of co-polymer was higher, greater extraction efficiencies could be reached. However, a larger amount of co-polymer would also require higher $\mathrm{HCl}$ concentrations (compared with the already selected concentration as optimum value) to ensure the formation of the hydrogel (since these variables are closely interconnected). Therefore, $10 \mathrm{mg}$ was selected as the optimum amount of co-polymer for the pH-HGME method, which is also an adequate amount for a microextraction method.

\subsection{Analytical performance of the entire pH-HGME-HPLC-FD method}

The entire pH-HGME-HPLC-FD method was validated, subjecting aqueous standards of the analytes to it. Table 1 includes some quality analytical parameters: calibration range, calibration slopes, correlation coefficients (R), standard deviation of the residuals $\left(\mathrm{S}_{\mathrm{y} / \mathrm{x}}\right)$, limits of detection (LODs) and limits of quantification (LOQs). LODs were determined by decreasing the concentration of the aqueous standards (under the application of the entire method) until a $\mathrm{S} / \mathrm{N}$ of 3 was obtained in the final chromatogram, while LOQs were estimated as $10 / 3$ the LODs and experimentally verified applying the entire method to aqueous standards prepared at those concentrations.

The calibration range was $0.1-3 \mu \mathrm{g} \mathrm{L}^{-1}$ for all the analytes, except for 20Hnap and nNP, for which the ranges were $0.3-3 \mu \mathrm{g} \mathrm{L}^{-1}$ and $0.5-5 \mu \mathrm{g} \mathrm{L}^{-1}$, respectively. The calibration sensitivity was maximum for $20 \mathrm{Hflu}$, while nNP presented the lowest sensitivity. The linearity of the method in the studied range was assessed using $R$ 
Table 2

Analytical performance of the entire pH-HGME-HPLC-FD method in terms of precision, extraction efficiency and relative recovery, using standard solutions in ultrapure water at two different concentration levels.

\begin{tabular}{|c|c|c|c|c|c|c|c|c|c|c|}
\hline \multirow{2}{*}{ Analyte } & \multicolumn{5}{|c|}{ Low concentration level $\left(0.60 \mu \mathrm{g} \mathrm{L}^{-1}\right)$} & \multicolumn{5}{|c|}{ High concentration level $\left(2.5 \mu \mathrm{g} \mathrm{L}^{-1}\right)$} \\
\hline & $\begin{array}{l}\text { Intra-day RSD } \\
\text { range }^{\mathrm{a}}(\%)\end{array}$ & $\begin{array}{l}\text { Intermediate } \\
\text { precision } \operatorname{RSD}^{\mathrm{b}}(\%)\end{array}$ & $\mathrm{E}_{\mathrm{F}}^{\mathrm{c}}$ & $E_{R}^{d}(\%)$ & $\mathrm{RR}^{\mathrm{e}}(\%)$ & $\begin{array}{l}\text { Intra-day RSD } \\
\text { range }^{\mathrm{a}}(\%)\end{array}$ & $\begin{array}{l}\text { Intermediate } \\
\text { precision } \mathrm{RSD}^{\mathrm{b}}(\%)\end{array}$ & $\mathrm{E}_{\mathrm{F}}^{\mathrm{c}}$ & $\mathrm{E}_{\mathrm{R}}{ }^{\mathrm{d}}(\%)$ & $\operatorname{RR}^{\mathrm{e}}(\%)$ \\
\hline 20Hnap & $5.4-7.5$ & 8.0 & 1.50 & 2.20 & 98.0 & $3.2-7.0$ & 9.2 & 2.30 & 3.40 & 98.1 \\
\hline 2OHflu & $4.6-6.5$ & 7.9 & 6.60 & 9.90 & 88.7 & $2.9-4.8$ & 6.8 & 9.00 & 13.6 & 96.3 \\
\hline 2OHphe & $4.1-5.4$ & 8.3 & 12.8 & 19.2 & 100 & $1.4-5.0$ & 6.5 & 14.2 & 21.3 & 94.6 \\
\hline Flu & $2.1-6.0$ & 7.1 & 10.8 & 16.2 & 99.2 & $2.4-6.7$ & 5.1 & 11.8 & 17.8 & 96.9 \\
\hline $\mathrm{Ft}$ & $6.1-7.9$ & 10 & 16.0 & 24.0 & 100 & $4.0-5.7$ & 6.4 & 17.7 & 26.6 & 96.0 \\
\hline $\mathrm{BaA}$ & $9.4-9.9$ & 16 & 13.7 & 20.5 & 89.1 & $1.9-5.1$ & 5.3 & 17.7 & 26.6 & 94.7 \\
\hline $\mathrm{nNP}$ & $4.1-8.6$ & 11 & 12.3 & 18.5 & 103 & $4.2-6.1$ & 11 & 9.90 & 14.9 & 86.7 \\
\hline
\end{tabular}

\footnotetext{
a Range (day 1 to day 3$)$ of relative standard deviation for intra-day precision $(n=3)$.

b Relative standard deviation for intermediate precision ( $n=12,3$ non-consecutive days).

c Enrichment factor.

d Extraction efficiency ( $\mathrm{E}_{\mathrm{Fmax}}$ 66.7).

e Relative recovery.
}

values, with values higher than 0.997 in all the cases. Moreover, a Student's $t$-test was carried out to verify the linearity [21].

As it can be observed in Table 1, the pH-HGME-HPLC-FD method characterizes for its high sensitivity, since LODs ranged from $1.0 \mathrm{ng} \mathrm{L}^{-1}$ for $20 \mathrm{Hflu}$ to $90 \mathrm{ng} \mathrm{L}^{-1}$ for nNP. These LOD values reveal the high preconcentration achieved with the microextraction procedure, particularly when compared with those obtained for the chromatographic method. Thus, LODs of the entire method were around 10 times lower than LODs of the HPLC-FD method for 2OHflu, Flu, and nNP and even more than 15 times lower for 2OHphe, Ft and BaA. It is also interesting to mention that, in general, the LODs were lower for the analytes with higher molecular weight within a specific family (see Table S1 of the ESM). In this sense, 20Hnap and Flu, as the representative analytes among the OH-PAHs and PAHs studied, presented LODs of $30 \mathrm{ng} \mathrm{L}^{-1}$ and $20 \mathrm{ng} \mathrm{L}^{-1}$, respectively.

The pH-HGME-HPLC-FD method was also evaluated in terms of precision, extraction efficiency, and relative recovery (RR). Table 2 includes some parameters of the analytical performance using aqueous standards at $0.60 \mu \mathrm{g} \mathrm{L}^{-1}$ as a low concentration level and $2.5 \mu \mathrm{g} \mathrm{L}^{-1}$ as a high concentration level. Both levels are included within the calibration range, but they were not used for the elaboration of calibration curves. Precision of the method was evaluated in intra-day and intermediate precision studies. Intra-day RSDs values were lower than $9.9 \%$ for the low concentration level. Regarding the RSD values for the intermediate precision, they were between $7.1 \%$ for Flu and $16 \%$ for BaA at the level of $0.60 \mu \mathrm{g} \mathrm{L}^{-1}$. According to the Horwitz equation [22], a maximum RSD of $32 \%$ is adequate for a concentration of $10 \mu \mathrm{g} \mathrm{L} \mathrm{L}^{-1}$, and even a RSD value of $45 \%$ is accepted for $1 \mu \mathrm{g} \mathrm{L}^{-1}$. Therefore, the precision of the method was acceptable given the concentration levels studied. The obtained RSD values are common in microextraction/miniaturized strategies (being the low amount of extraction material the main source of error, together with issues associated to possible dilution of the hydrogel-rich phase if the aqueous phase is not properly removed in the final step). Regarding the RR values, they were close to $100 \%$ for most of the analytes, with average values of $96.9 \%$ and 94.8\% at the low and high concentration level, respectively.

The preconcentration of the pH-HGME-HPLC-FD method was assessed with the $E_{F}$ values, calculated as described in Section 3.2.1. Thus, $\mathrm{E}_{\mathrm{F}}$ values included in Table 2 ranged from 1.50 for 2OHnap at the low concentration level to $17.7 \mathrm{for} F t$ and BaA at the high concentration level. The preconcentration achieved can be clearly observed if comparing the chromatograms shown in Fig. 3(A) and (B).

Extraction efficiencies $\left(E_{R}, \%\right)$ were calculated as the ratio between $E_{F}$ and the theoretical maximum enrichment factor $\left(E_{F m a x}\right)$.
$\mathrm{E}_{\mathrm{Fmax}}$ was estimated as the ratio between the initial volume of the aqueous standard and the volume of the final extract subjected to analysis ( $\sim 150 \mu \mathrm{L}$ after dissolving the hydrogel-rich phase in $\mathrm{ACN})$. Thus, $\mathrm{E}_{\mathrm{Fmax}}$ was $\sim 66.7$. The $\mathrm{E}_{\mathrm{R}}$ values showed in Table 2 were between $2.20 \%$ for 20 Hnap at the low concentration level and $26.6 \%$ for Ft and $\mathrm{BaA}$ at the high concentration level. It is important to mention that in analytical microextraction approaches, $\mathrm{E}_{\mathrm{R}}$ values lower than $20 \%$ are acceptable, as long as the method is reproducible, it achieves good preconcentration, and it has adequate sensitivity for the intended application [23]. Therefore, the $E_{R}$ values of the pH-HGME-HPLC-FD method were adequate for all the analytes, except for 20Hnap and 20Hflu.

In general, the $E_{R}$ values for the PAHs were higher than those obtained for the group of OH-PAHs. This may be related to the $\mathrm{pH}$ required for the solubilization of the $\mathrm{pH}$-sensitive polymer. The basic medium in which the microextraction is initially performed favors the formation of the alkoxides of the OH-PAHs, and increases the solubility of these analytes in water, thus reducing their interaction with the polymer (and ultimately with the hydrogel formed) in the aqueous medium. It is important to highlight that all these interpretations are preliminary assumptions of the possible affinity of the polymer (and afterwards the hydrogel) towards analytes with different structures with the aim for a better understanding of the interactions between the polymer and the analytes. However, deeper studies with a larger number of analytes are needed to obtain definitive conclusions about the influence of the $\mathrm{pH}$ and the nature of the analytes on the effectiveness of the $\mathrm{pH}$-sensitive polymer used as extraction medium.

At this point, it is interesting to compare the pH-HGME-HPLCFD method with other methods described in the recent literature. Table S7 of the ESM shows the several parameters of different extraction methods that have been used in combination with HPLCFD for the determination of some of the analytes studied in the current study. Thus, different novel materials have been used to extract these analytes, such as ionic liquids (or derivatives) in liquid phase microextraction strategies $[17,24,25]$ and magnetic sorbents in solid phase extraction methods [26]. The amount of extraction material used in the pH-HGME method is similar to that reported in the methods listed in Table S7 of the ESM, for which small volumes (around $\mu \mathrm{L}$ ) of extraction solvent $[17,24,25]$ or a few mg of sorbent were used [26]. In addition, the proposed extraction method takes around $20 \mathrm{~min}$, a similar time to other microextraction methods, for which times of $\sim 30$ min have been reported $[25,26]$. The $\mathrm{pH}-\mathrm{HGME}-\mathrm{HPLC}-\mathrm{FD}$ method presented LODs at the ng $\mathrm{L}^{-1}$ level for the studied analytes, as well as the other methods included in Table S7 of the ESM, which reported LODs ranging from $0.75 \mathrm{ng} \mathrm{L}^{-1}$ for $\mathrm{BaA}$ [26] to $350 \mathrm{ng} \mathrm{L}^{-1}$ for $\mathrm{nNP}$ [25] in water 
Table 3

Analytical performance of the pH-HGME-HPLC-FD method in real urine samples, including quality analytical parameters of the standard addition calibration method with hydrolyzed urine, as well as the predicted concentrations in the samples.

\begin{tabular}{|c|c|c|c|c|c|c|c|c|}
\hline \multirow{2}{*}{ Analyte } & \multicolumn{4}{|c|}{ Non-smoker male urine } & \multicolumn{4}{|c|}{ Smoker female urine } \\
\hline & (Slope \pm SD $^{\mathrm{a}}$ ) & $\mathrm{R}^{\mathrm{b}}$ & $\mathrm{S}_{\mathrm{y} / \mathrm{x}}{ }^{\mathrm{c}}$ & $\begin{array}{l}\text { Predicted conc. }^{\mathrm{d}} \pm \mathrm{S}_{\mathrm{XE}}{ }^{\mathrm{e}} \\
\left(\mu \mathrm{g} \mathrm{L}^{-1}\right)\end{array}$ & (Slope $\pm \mathrm{SD}^{\mathrm{a}}$ ) & $\mathrm{R}^{\mathrm{b}}$ & $\mathrm{S}_{\mathrm{y} / \mathrm{x}}{ }^{\mathrm{c}}$ & $\begin{array}{l}\text { Predicted conc. }{ }^{d} \pm S_{X E}{ }^{e} \\
\left(\mu \mathrm{g} \mathrm{L}^{-1}\right)\end{array}$ \\
\hline 20Hnap & $22 \pm 1$ & 0.996 & 1.9 & $7.3 \pm 0.4$ & $23 \pm 4$ & 0.996 & 2.7 & $19.3 \pm 0.6$ \\
\hline 2OHflu & $154 \pm 3$ & 0.999 & 7.3 & n.d. & $139 \pm 1$ & 0.999 & 8.2 & n.d. \\
\hline 20Hphe & $123 \pm 3$ & 0.999 & 6.4 & n.d. & $122 \pm 4$ & 0.998 & 9.0 & n.d. \\
\hline Flu & $128 \pm 6$ & 0.997 & 14.8 & n.d. & $118 \pm 4$ & 0.998 & 9.8 & n.d. \\
\hline $\mathrm{Ft}$ & $38 \pm 1$ & 0.999 & 2.3 & n.d. & $38 \pm 1$ & 0.999 & 2.4 & n.d. \\
\hline $\mathrm{BaA}$ & $97 \pm 2$ & 0.999 & 5.3 & n.d. & $77 \pm 3$ & 0.998 & 5.9 & n.d. \\
\hline $\mathrm{nNP}$ & $4.5 \pm 0.2$ & 0.997 & 0.5 & n.d. & $4.0 \pm 0.1$ & 0.999 & 0.3 & n.d. \\
\hline
\end{tabular}

n.d.: non-detected.

a Standard deviation of the slope within the calibration range $\left(0-3 \mu \mathrm{L} \mathrm{L}^{-1}\right.$ for all the analytes; $\left.n=5\right)$ for a significance level of $5 \%$.

b Correlation coefficient.

c Standard deviation of the residuals.

d Concentration obtained after applying the dilution factor to the urine samples.

e Uncertainty in the prediction of the concentration, calculated with the following equation: $S_{X E}=\frac{S_{y / x}}{b} \cdot \sqrt{\frac{1}{n}+\frac{\bar{y}^{2}}{b^{2} \cdot \sum_{i}\left(x_{i}-\bar{x}\right)^{2}}}$.

samples. Regarding the extraction efficiency, the $E_{R}$ values reported for other methods are close to those obtained in the present study $[17,24]$. In short, the pH-HGME method provides comparable results to other strategies reported in the literature but with the advantage of using a smart material that is dispersed in the sample due to its solubility in water. Furthermore, the process also characterizes by its sustainability, taking into account the biocompatibility of the pH-sensitive polymer and the requirement of a simple $\mathrm{pH}$ change to induce its separation from the sample.

\subsection{Application of the pH-HGME-HPLC-FD method to the analysis of urine samples}

The pH-HGME-HPLC-FD method was applied to the determination of the seven organic compounds in real samples. To the best of our knowledge, these groups of analytes have not been determined simultaneously; despite the occurrence of all of them in urine samples has been described [27-29]. The current human exposure to PAHs and nNP is of special interest and concern, taking into account their toxic effects as carcinogenic agents and endocrine disruptors $[27,30]$. In the case of PAHs, their urinary concentration is low and the monitoring of their urinary metabolites, which requires a previous hydrolysis step to generate the OH-PAH form, is preferred to evaluate the exposure to these toxic compounds [31]. In the light of this background, human urine was selected as the matrix to evaluate the applicability of the method for the analysis of real samples.

Two urine samples from different individuals were used: a nonsmoker male and a smoker female. They were selected with comparative purposes since, a priori, there might be found different concentrations of some of the analytes depending on the smoking habits and other lifestyle factors of the individuals [31].

Precision and RR studies were carried out using non-hydrolyzed urine samples spiked at $0.60 \mu \mathrm{g} \mathrm{L}^{-1}$. The urine samples were not subjected to the hydrolysis step for this study to minimize the presence of the OH-PAHs and evaluate the effect of other matrix components on the analytical performance of the method. The precision was assessed as intra-day $\operatorname{RSD}(n=3)$, showing values that ranged from $3.7 \%$ to $9.0 \%$ for the non-smoker urine and from $4.4 \%$ to $8.6 \%$ for the smoker urine. Thus, the method exhibited highly adequate precision in urine despite the low spiked level used and the complexity of the matrix. Regarding the RR values, they were always higher than $76.5 \%$ for both urine samples. However, in some cases RR were higher than $120 \%$, demonstrating several differences between the values obtained in urine and those obtained in ultrapure water at the spiked level of $0.60 \mu \mathrm{L} \mathrm{L}^{-1}$ for some of the analytes (see Table 2), which may be an indicative that the matrix has an effect on the performance of the method.

With the aim of simultaneously assessing the matrix effect of the pH-HGME-HPLC-FD method and determining the analytes in the urine samples, standard addition calibration curves were performed in hydrolyzed urine samples. Several quality analytical parameters of these calibration curves are shown in Table 3 . $R$ values were higher than 0.996 for both samples. Regarding the calibration slopes, a statistical test described by Andrade et al. [32] was carried out to evaluate the matrix effect by the comparison between the slopes of the standard addition calibrations in urine and those of the calibrations in ultrapure water. Table S8 of the ESM shows the main statistical parameters. The results of the Student's $t$-test confirmed that there is matrix effect for 2OHnap, $20 \mathrm{Hflu}$ and nNP in the non-smoker male urine, and for 2OHnap, BaA and nNP in the smoker female urine. Therefore, the use of standard addition method is advisable for the determination of those analytes in urine samples. In any case, it is interesting to highlight that the spiked analytes can be easily detected in the urine samples using the pH-HGME-HPLC-FD method despite the high complexity of this matrix. Fig. 3(C) and Fig. 3(D) show representative chromatograms obtained after applying the pH-HGME-HPLC-FD method to both hydrolyzed urine samples. It can be observed that there are not signals coming from the matrix that interfere with the determination of the analytes, except for the signals associated to the sample in the first $3 \mathrm{~min}$ of the chromatograms.

The standard addition calibrations with the entire pH-HGMEHPLC-FD method were used for the quantification of the analytes in the analyzed urine samples. Predicted concentrations are shown in Table 3, whereas Table S9 of the ESM includes the intercepts for each analyte, as well as the probability level to have those intercepts equal or different to zero according to a Student's $t$-test. 2OHnap was the only analyte quantified in both samples, with a concentration of $7.3 \pm 0.4 \mu \mathrm{g} \mathrm{L}^{-1}$ in the non-smoker male urine, and $19.3 \pm 0.6 \mu \mathrm{g} \mathrm{L}^{-1}$ in the smoker female urine. These results agree with the smoking habits of the individuals since higher urinary concentrations of these analytes are normally found in smokers [31]. In any case, LC-MS is advisable to confirm the identification of the analytes in the urine due to the high complexity of the sample.

\section{Conclusions}

A simple microextraction approach using a pH-sensitive polymer (pH-HGME) is developed successfully. The method simply takes advantage on the in situ generation of a hydrogel in the 
aqueous sample at low $\mathrm{pH}$ values. The current method directly combines with HPLC-FD, being the first application in which a hydrogel-rich final extract does not require an additional backextraction step prior to the analysis.

An experimental design facilitated the optimization of the entire pH-HGME-HPLC-FD method for the determination of seven organic compounds, including PAHs, OH-PAHs and an alkylphenol. The validation in ultrapure water showed satisfactory intermediate precision, high preconcentration and sensitivity, with LODs at the ng $\mathrm{L}^{-1}$ level. The method was also able to succeed with complex samples such as human urine, in which 20Hnap was quantified at low concentrations using the standard addition method.

The incorporation of the $\mathrm{pH}$-sensitive polymer in a microextraction approach led to the development of a simple, fast and efficient analytical method due to the stimuli-responsive characteristics of this material. Furthermore, the high biocompatibility of the polymer makes it a promising material to be used as extraction medium in bioanalytical methods, which can even expand to in vivo applications. Ongoing work is focused on a better understanding of the physicochemical interactions between the $\mathrm{pH}$-sensitive polymer and the analytes in order to design new polymers that allow maximization of the extraction efficiencies and ultimately to develop more sensitive and selective analytical sample preparation methods.

\section{Declaration of Competing Interest}

The authors declare that they have no known competing financial interests or personal relationships that could have appeared to influence the work reported in this paper.

\section{CRediT authorship contribution statement}

Raúl González-Martín: Formal analysis, Investigation, Methodology, Writing - original draft, Validation. Idaira PachecoFernández: Formal analysis, Investigation, Methodology, Writing original draft, Validation. Binoy Maiti: Investigation, Methodology. Juan H. Ayala: Software, Supervision, Validation. Ana M. Afonso: Supervision, Validation, Methodology, Writing - review \& editing. David Díaz Díaz: Investigation, Methodology, Supervision, Writing - review \& editing. Verónica Pino: Investigation, Methodology, Funding acquisition, Project administration, Resources, Supervision, Writing - review \& editing.

\section{Acknowledgments}

I. P.-F. thanks the Canary Agency of Research and Innovation (ACIISI), co-funded by the European Social Fund, for her FPI PhD fellowship. B. M. and D. D. D. thank the University of Regensburg for financial support. D. D. D. also thanks the Spanish Ministry of Science, Innovation and Universities for the Senior "Beatriz Galindo" Distinguished Researcher Award. V. P. thanks the Spanish Ministry of Economy and Competitiveness (MINECO) for the project MAT2017-89207-R. The authors extend their appreciation to the Spanish Ministry of Science, Innovation and Universities for granting the Spanish Network of Excellence in Sample Preparation (RED2018-102522-T). This article is based upon work from the Sample Preparation Task Force and Network supported by the Division of Analytical Chemistry of the European Chemical Society.

\section{Supplementary materials}

Supplementary material associated with this article can be found, in the online version, at doi:10.1016/j.chroma.2020.460910.

\section{References}

[1] J. Brijitta, P. Schurtenberger, Responsive hydrogel colloids: structure, interactions, phase behavior, and equilibrium and nonequilibrium transitions of microgel dispersions, Curr. Opin. Colloid Interface Sci. 40 (2019) 87-103.

[2] J. Jiao, J. Huang, Z. Zhang, Hydrogels based on chitosan in tissue regeneration: how do they work? a mini review, J. Appl. Polym. Sci. 136 (2018) 47235.

[3] S. Chatterjee, P.C. Hui, C. Kan, Thermoresponsive hydrogels and their biomedical applications: special insight into their applications in textile based transdermal therapy, Polymers (Basel) 10 (2018) 480.

[4] O. Erol, A. Pantula, W. Liu, D.H. Gracias, Transformer hydrogels: a review, Adv. Mater. Technol. 4 (2019) 1900043.

[5] R. Liang, L. Wang, H. Yu, A. Khan, B.U. Amin, R.U. Khan, Molecular design, synthesis and biomedical applications of stimuli-responsive shape memory hydrogels, Eur. Polym. J. 114 (2019) 380-396.

[6] W. Cheng, Y. Liu, Redox-responsive hydrogels, in: S. Jana, S. Maiti, S. Jana (Eds.), Biopolymer-based composites: Drug Delivery and Biomedical Applications, Woodhead Publishing, Cambridge, 2017, pp. 31-60.

[7] J. Shang, P. Theato, Smart composite hydrogel with $\mathrm{pH}-$, ionic strength- and temperature-induced actuation, Soft Matter 14 (2018) 8401-8407.

[8] Q. Yan, L. Liu, T. Wang, H. Wang, A pH-responsive hydrogel system based on cellulose and dopamine with controlled hydrophobic drug delivery ability and log-term bacteriostatic property, Colloid Polym. Sci. 297 (2019) 705-717.

[9] H. Khan, J.P. Chaudhary, R. Meena, Anionic carboxymethylagarose-based pH-responsive smart superabsorbent hydrogels for controlled release of anticancer drug, Int. J. Biol. Macromol. 124 (2019) 1220-1229.

[10] G.S. Longo, N.A. Pérez-Chávez, I. Szleifer, How protonation modulates the interaction between proteins and pH-responsive hydrogel films, Curr. Opin. Colloid Interface Sci. 41 (2019) 27-39.

[11] M. Bahram, F. Keshvari, P. Najafi-Moghaddam, Development of cloud point extraction using $\mathrm{pH}$-sensitive hydrogel for preconcentration and determination of malachite green, Talanta 85 (2011) 891-896.

[12] M. Bahram, N. Nurallahzadeh, N. Mohseni, pH-sensitive hydrogel for coacervative cloud point extraction and spectrophotometric determination of $\mathrm{Cu}$ (II): optimization by central composite design, J. Iran Chem. Soc. 12 (2015) 1781-1787.

[13] X. Chen, Z. Guo, Y. Wang, Y. Liu, Y. Xu, J. Liu, Z. Li, J. Zhao, Temperature sensitive polymer-dispersive liquid-liquid microextraction with gas chromatography-mass spectrometry for the determination of phenols, J. Chromatogr. A 1592 (2019) 183-187.

[14] H. Yu, Z. Wang, R. Wu, X. Chen, T.-W- D. Chan, Water-dispersible pH/thermo dual-responsive microporous polymeric microspheres as adsorbent for dispersive solid-phase extraction of fluoroquinolones from environmental water samples and food samples, J. Chromatogr. A 1601 (2019) 27-34.

[15] N.M.F.M. Sampaio, N.D.B. Castilhos, B.C. da Silva, I.C. Riegel-Vidotti, B.J.G. Silva, Evaluation of polyvinyl alcohol/pectin-based hydrogel disks as extraction phase for determination of steroidal hormones in aqueous samples by GC-MS/MS, Molecules 24 (2019) 40.

[16] S. Armenta, S. Garrigues, F.A. Esteve-Turrillas, M. de la Guardia, Green extraction techniques in green analytical chemistry, Trac-Trends Anal. Chem. 116 (2019) 248-253.

[17] I. Pacheco-Fernández, V. Pino, J. Lorenzo-Morales, J.H. Ayala, A.M. Afonso, Salt-induced ionic liquid-based microextraction using a low cytotoxic guanidinium ionic liquid and liquid chromatography with fluorescence detection to determine monohydroxylated polycyclic aromatic hydrocarbons in urine, Anal. Bioanal. Chem. 410 (2018) 4701-4713.

[18] S.M. Henry, M.E.H. El-Sayed, C.M. Pirie, A.S. Hoffman, P.S. Stayton, pH-Responsive poly(styrene-alt-maleic anhydride) alkylamide copolymers for intracellular drug delivery, Biomacromolecules 7 (2006) 2407-2414.

[19] X. Duan, J. Xiao, Q. Yin, Z. Zhang, S. Mao, Y. Li, Amphiphilic graft copolymer based on poly(styrene-co-maleic anhydride) with low molecular weight polyethylenimine for efficient gene delivery, Int. J. Nanomed. 7 (2012) 4961-4972.

[20] S.L.C. Ferreira, W.N.L. dos Santos, C.M. Quintella, B.B. Neto, J.M. Bosque-Sendra, Doehlert matrix: a chemometric tool for analytical chemistry-review, Talanta 63 (2004) 1061-1067.

[21] J.N. Miller, J.C. Miller, Statistics and Chemometrics for Analytical Chemistry, 6th ed., Pearson, Harlow, 2010.

[22] I. Traverniers, M. De Loose, E. Van Bockstaele, Trends in quality in the analytical laboratory. II. analytical method validation and quality assurance, Trac-Trends Anal. Chem. 23 (2004) 535-552.

[23] M.J. Trujillo-Rodríguez, P. Rocío-Bautista, V. Pino, A.M. Afonso, Ionic liquids in dispersive liquid-liquid microextraction, Trac-Trends Anal. Chem. 51 (2013) 87-106.

[24] M.J. Trujillo-Rodríguez, O. Nacham, K.D. Clark, V. Pino, J.L. Anderson, J.H. Ayala, A.M. Afonso, Magnetic ionic liquids as non-conventional extraction solvents for the determination of polycyclic aromatic hydrocarbons, Anal. Chim. Acta 934 (2016) 106-113

[25] Q. Zhou, Y. Gao, G. Xie, Determination of bisphenol A, 4-n-nonylphenol, and 4-tert-octylphenol by temperature-controlled ionic liquid dispersive liquid-phase microextraction combined with high performance liquid chromatography-fluorescence detector, Talanta 85 (2011) 1598-1602.

[26] X. Yang, Y. Yin, Y. Zong, T. Wan, X. Liao, Magnetic nanocomposite as sorbent for magnetic solid phase extraction coupled with high performance liquid chromatography for determination of polycyclic aromatic hydrocarbons, Microchem. J. 145 (2019) 26-34. 
[27] I.-.H. Acir, K. Guenther, Endocrine-disrupting metabolites of alkylphenol ethoxylates - A critical review of analytical methods, environmental occurrences, toxicity, and regulation, Sci. Total Environ. 635 (2018) 1530-1546.

[28] M. Martinefski, N. Feizi, M.L. Lunar, S. Rubio, Supramolecular solvent-based high-throughput sample treatment platform for the biomonitoring of PAH metabolites in urine by liquid chromatography-tandem mass spectrometry, Chemosphere 237 (2019) 124525.

[29] E. Moradi, H. Ebrahimzadeh, Z. Mehrani, Electrospun acrylonitrile butadiene styrene nanofiber film as an efficient nanosorbent for head space thin film microextraction of polycyclic aromatic hydrocarbons from water and urine samples, Talanta 205 (2019) 120080.
[30] I. Abbas, G. Badran, A. Verdin, F. Ledoux, M. Roumié, D. Courcot, G. Garçon, Polycyclic aromatic hydrocarbon derivatives in airborne particulate matter: sources, analysis and toxicity, Environ. Chem. Lett. 16 (2018) 439-475.

[31] B.-C. Yang, S.-F. Fang, X.-J. Wan, Y. Luo, J.-Y. Zhou, Y. Li, Y.-J. Li, F. Wang, O.-P. Huang, Quantification of monohydroxylated polycyclic aromatic hydrocarbons in human urine samples using solid-phase microextraction coupled with glass-capillary nanoelectrospray ionization mass spectrometry, Anal. Chim. Acta 973 (2017) 68-74.

[32] J.M. Andrade, M.G. Estévez-Pérez, Statistical comparison of the slopes of two regression lines: a tutorial, Anal. Chim. Acta 838 (2014) 1-12. 